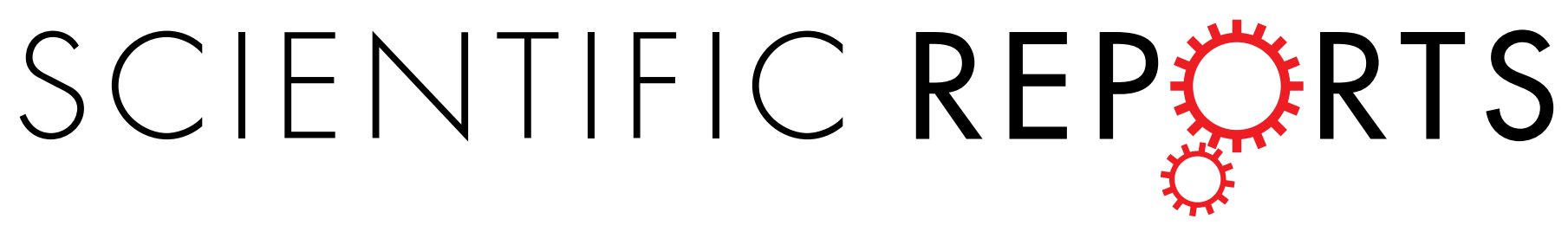

\title{
Erratum: A sensitised RNAi screen reveals a ch-TOG genetic interaction network required for spindle assembly
}

\section{Alexis R. Barr \& Chris Bakal}

Scientific Reports 5:10564; doi: 10.1038/srep10564; published online 03 June 2015; updated on 31 July 2015 In the original version of this Article, the Abstract contains a typographical error.

"We identified a ch-TOG centred network of genetic interactions which promotes ensures centrosome-mediated microtubule polymerisation, leading to the incorporation of microtubules polymerised by all pathways into a bipolar structure."

should read:

"We identified a ch-TOG centred network of genetic interactions which promotes centrosome-mediated microtubule polymerisation, leading to the incorporation of microtubules polymerised by all pathways into a bipolar structure."

This has now been corrected in both the PDF and HTML versions of the Article. 